\title{
Investigation of Ion Generation Rates in an Inertial Electrostatic Confinement Device by Spectroscopy-Based Inverse Analysis
}

\author{
Tomonobu ITAGAKI, Jun HASEGAWA and Eiki HOTTA \\ Tokyo Institute of Technology, Tokyo 152-8550, Japan
}

(Received 3 July 2020 / Accepted 11 August 2020)

\begin{abstract}
A novel inverse analysis method was developed and tested to determine the spatial distribution of the ionization rate of hydrogen gas in an inertial electrostatic confinement (IEC) fusion device. The ionization rate distributions were inversely determined so that an experimentally observed $\mathrm{H}_{\alpha}$ spectrum could be reproduced by the linear combination of $\mathrm{H}_{\alpha}$ partial spectra that were numerically predicted by one-dimensional Monte Carlo simulations. This method is useful for improving the performance of IEC fusion device as a neutron source because the ionization rate distribution greatly affects the ion energy distribution and fusion rate.
\end{abstract}

(c) 2020 The Japan Society of Plasma Science and Nuclear Fusion Research

Keywords: inertial electrostatic confinement fusion, ion Doppler spectroscopy, Monte Carlo simulation

DOI: $10.1585 /$ pfr. 15.1206070

This paper presents a novel analysis method that can indirectly determine the spatial distribution of ionization rate in the inertial electrostatic confinement (IEC) fusion device using an inverse analysis technique. The method uses a one-dimensional Monte Carlo (MC) simulation to predict a series of partial $\mathrm{H}_{\alpha}$ emission spectra originating from deuterium/hydrogen ions that are initially generated at different locations in the IEC device. Then the ionization rate distribution is inversely determined so that an experimentally observed $\mathrm{H}_{\alpha}$ spectrum can be reproduced by a linear combination of the numerically obtained partial spectra.

Figure 1 shows a schematic of the linear IEC device used in this study. A hollow cathode $(\varnothing 6 \times 10 \mathrm{~cm})$ and two facing anodes $(\varnothing 2 \times 6 \mathrm{~cm})$ made of stainless steel are arranged coaxially on the center axis of the device with gap lengths of $115 \mathrm{~mm}$. The electrical insulation between the cathode and anodes is established by borosilicate-glass tubes with an inner diameter of $134 \mathrm{~mm}$. Hydrogen gas was supplied through a flow control valve $(\sim 2 \mathrm{sccm})$ and evacuated by a turbo molecular pump through a throttle valve. The gas pressure was stably maintained at $\sim 1$ Pa by the feedback control of the throttle valve. The cathode was negatively biased up to $-30 \mathrm{kV}$ by a high-voltage power supply to induce glow discharge in the gas. The power supply was operated with a constant current up to $30 \mathrm{~mA}$, and the cathode voltage was manually controlled by the gas pressure.

The light emitted from the discharge column was extracted through the anode center hole and then focused by a lens onto the inlet of an optical fiber connected to a 50cm monochromator. The spectral shape of $\mathrm{H}_{\alpha}$ emission was recorded by a high-resolution EM-CCD camera. As

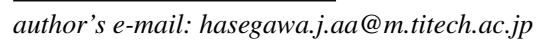

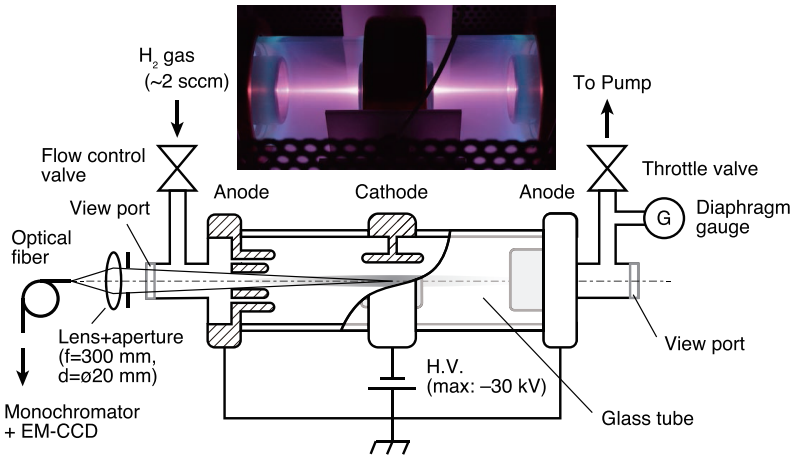

Fig. 1 Experimental setup for emission spectroscopy with a glass-wall linear IEC device.

shown in Fig. 1, a bright column was locally formed during the discharge along the center axis of the device, which indicated that the ionization reactions of hydrogen gas occurred in the limited volume near the center axis of the device.

A one-dimensional Monte Carlo (MC) code was developed to simulate the motions of hydrogen particles along the center axis of the device and calculate their distribution functions $f_{i}(x, v)$ after various collisional interactions. Here, $x$ and $v$ are, respectively, the position and velocity of hydrogen atoms that eventually cause $\mathrm{H}_{\alpha}$ emission. Suffix $i$ denotes the species of parent particles such as $\mathrm{H}^{+}, \mathrm{H}_{2}^{+}, \mathrm{H}_{3}^{+}, \mathrm{H}$, and $\mathrm{H}_{2}$, which dissociate into excited hydrogen atoms $(n=3)$ after charge exchange $(\mathrm{CX})$ reactions with $\mathrm{H}_{2}$ molecules. The code considered the reactions listed in Table 1 with the cross sections complied by Tabata et al.[1].

An electrostatic potential along the center axis of the device [dotted line in Fig. 2(a)] was calculated by a finite 
Table 1 Collisional reactions considered in the developed onedimensional MC code. No excited states are considered.

\begin{tabular}{lll}
\hline Projectiles & Reactions \\
\hline $\mathrm{H}^{+}$ & $\mathrm{H}^{+}+\mathrm{H}_{2} \rightarrow$ fast $\mathrm{H}, \quad \mathrm{H}^{+}+\mathrm{H}_{2} \rightarrow \mathrm{H}_{\alpha}$ \\
\hline $\mathrm{H}_{2}^{+}$ & $\mathrm{H}_{2}^{+}+\mathrm{H}_{2} \rightarrow \mathrm{H}_{3}^{+}+\mathrm{H}, \quad \mathrm{H}_{2}^{+}+\mathrm{H}_{2} \rightarrow$ slow $\mathrm{H}_{2}^{+}(*)$, \\
& $\mathrm{H}_{2}^{+}+\mathrm{H}_{2} \rightarrow$ fast $\mathrm{H}^{+}, \quad \mathrm{H}_{2}^{+}+\mathrm{H}_{2} \rightarrow \mathrm{H}_{\alpha}$ \\
\hline $\mathrm{H}_{3}^{+}$ & $\mathrm{H}_{3}^{+}+\mathrm{H}_{2} \rightarrow$ fast $\mathrm{H}^{+}, \quad \mathrm{H}_{3}^{+}+\mathrm{H}_{2} \rightarrow$ fast $\mathrm{H}_{2}^{+}$, \\
& $\mathrm{H}_{3}^{+}+\mathrm{H}_{2} \rightarrow$ fast $\mathrm{H}, \quad \mathrm{H}_{3}^{+}+\mathrm{H}_{2} \rightarrow$ fast $\mathrm{H}_{2}$, \\
& $\mathrm{H}_{3}^{+}+\mathrm{H}_{2} \rightarrow \mathrm{H}_{\alpha}$ \\
\hline $\mathrm{H}$ & $\mathrm{H}+\mathrm{H}_{2} \rightarrow$ slow $\mathrm{H}_{2}^{+}, \quad \mathrm{H}+\mathrm{H}_{2} \rightarrow$ fast $\mathrm{H}^{+}$, \\
& $\mathrm{H}+\mathrm{H}_{2} \rightarrow \mathrm{H}_{\alpha}$ \\
\hline $\mathrm{H}_{2}$ & $\mathrm{H}_{2}+\mathrm{H}_{2} \rightarrow$ fast $\mathrm{H}_{2}^{+}, \quad \mathrm{H}_{2}+\mathrm{H}_{2} \rightarrow$ fast $\mathrm{H}^{+}$, \\
& $\mathrm{H}_{2}+\mathrm{H}_{2} \rightarrow \mathrm{H}_{\alpha}$ \\
\hline
\end{tabular}

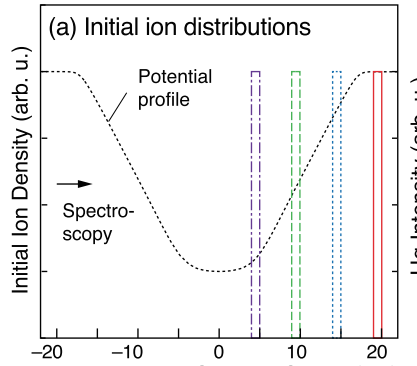

Distance from Cathode Center $(\mathrm{cm})$
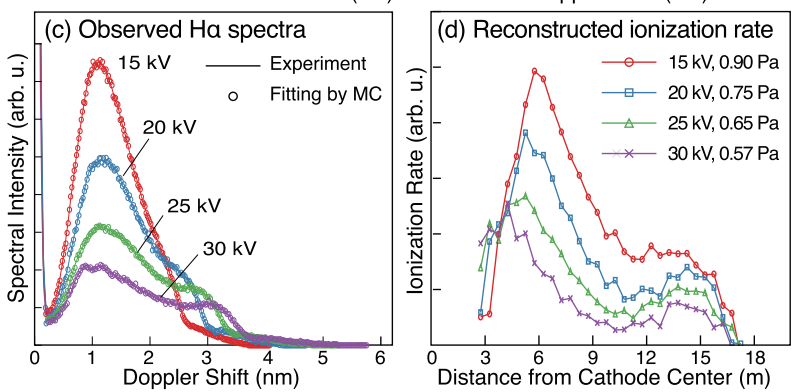

Fig. 2 Typical results of MC simulations and emission spectroscopy on $\mathrm{H}_{\alpha}$ Doppler components and reconstructed ionization results under various discharge conditions.

element method and then imported to the MC code. Because the hydrogen gas in the IEC device was weakly ionized, space-charge and Debye-shielding effects were ignored here. It was also assumed that only $\mathrm{H}_{2}^{+}$were initially generated because the cross sections of $\mathrm{H}^{+}$production from $\mathrm{H}_{2}$ owing to electron impact and charge transfer are relatively small.

Typical $\mathrm{H}_{\alpha}$ emission spectra calculated by the MC simulation are shown in Fig. 2 (b). Each partial spectrum was obtained by assuming that $\mathrm{H}_{2}^{+}$ions were initially generated only in a limited space $(\Delta x=5 \mathrm{~mm})$ shown by rectangles in Fig. 2 (a). In each case, $10^{6}$ trials were accumulated to obtain sufficient statistical accuracy. The spectra calculated in Fig. 2 (b) have both blue- (left) and red-
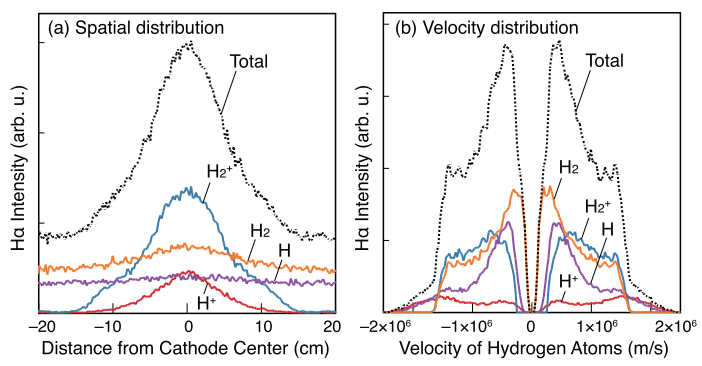

Fig. 3 Spatial and velocity distribution functions of $\mathrm{H}_{\alpha}$-emitting hydrogen particles calculated using ion production rate distribution obtained by the inverse analysis.

shifted Doppler peaks (right). The large asymmetry in these spectra means that most hydrogen ions caused $\mathrm{H}_{\alpha}$ emission via $\mathrm{CX}$ and dissociative reactions in their first flight toward the cathode. This is supported by the fact that the mean free path of $30-\mathrm{keV} \mathrm{H}_{2}^{+}$ions in $0.56-\mathrm{Pa} \mathrm{H}_{2}$ gas estimated from the cross section of the CX reaction ( $*$ in Table 1 ) is only $\sim 10 \mathrm{~mm}$. The positions of sharp peaks in the partial spectra move to the smaller Doppler-shift side as the location of the initial ion generation becomes closer to the cathode center. This occurs because the CX reactions causing $\mathrm{H}_{\alpha}$ emission are more likely to occur for faster parent ions in an energy regime of $\sim 30 \mathrm{keV}$. Assuming that the observed $\mathrm{H}_{\alpha}$ spectrum $S_{\text {exp }}(\lambda)$ is expressed by the linear combination of partial $\mathrm{H}_{\alpha}$ spectra $S_{j}(\lambda)$ obtained by MC simulation, the following equation should be established: $S_{\exp }(\lambda)=\sum_{j=1}^{m} g_{j} S_{j}(\lambda)$. Here, $g_{j}$ is a weighting coefficient for the partial spectrum $S_{j}(\lambda)$ that originates from the ions generated in the $j$ th region in the calculation field. Figure 2(c) shows numerically reconstructed $\mathrm{H}_{\alpha}$ emission spectra (open circles), which were obtained by solving the weighting factor $g_{j}$ using the least square method. The reconstructed spectra well reproduce the experimental ones (solid lines). In Fig. 2 (d), the weighting factor $g_{j}$ is plotted as a function of location where hydrogen ions are initially produced. The spatial profile of $g_{j}$ has a large peak near the cathode and a small one near the anode, which indicates that most of $\mathrm{H}_{2}^{+}$ions are generated by charge exchange reactions between $\mathrm{H}_{2}^{+}$and $\mathrm{H}_{2}$ near the anode and by electron impact ionization of $\mathrm{H}_{2}$ near the cathode.

Figure 3 shows the spatial and velocity distributions of $\mathrm{H}_{\alpha}$ emitting hydrogen atoms calculated using the ionization rate distribution $(30 \mathrm{kV}$ and $0.57 \mathrm{~Pa})$ shown in Fig. 2 (d). The solid lines show the $\mathrm{H}_{\alpha}$ emission originating from specific "parent" hydrogen particles, and the dotted lines represent their total distribution. As shown in Fig. 3 (a), the $\mathrm{H}_{\alpha}$ emission becomes maximum at the center of the cathode owing to the contributions of $\mathrm{H}_{2}^{+}$and $\mathrm{H}^{+}$, having the highest $\mathrm{CX}$ probabilities at the maximum energy $(\sim 30 \mathrm{keV})$. In contrast, the contributions of neutral particles are almost uniform. Figure 3(b) shows that a large peak in $\mathrm{H}_{\alpha}$ spectra with relatively small Doppler shifts are 
mainly due to the neutral hydrogen molecules and atoms. With an increase in the Doppler shift, the contributions of hydrogen ions $\left(\mathrm{H}_{2}^{+}\right.$and $\left.\mathrm{H}^{+}\right)$relatively increases; however fast $\mathrm{H}_{2}$ molecules are still essential in the $\mathrm{H}_{\alpha}$ emission process. This result shows that $\mathrm{CX}$ reactions between fast $\mathrm{H}_{2}^{+}$ ions and slow $\mathrm{H}_{2}$ molecules are predominant in the IEC discharge.

In conclusion, the developed inverse analysis method was successfully applied to determine ionization rate dis- tributions in the IEC device from the observed $\mathrm{H}_{\alpha}$ spectra. Because the MC code can easily include fusion reactions, this method will be also used to predict neutron production rates in deuterium gas discharge and improve the performance of IEC fusion devices.

[1] T. Tabata and T. Shirai, At. Data Nucl. Data Tables, 76, 1 (2000). 